\title{
Second-Generation Platelet Concentrates (PRFs): Estimation of Cellular Content
}

\author{
Alessandro Crisci ${ }^{1,2,3}, *$ (D) , Michela Crisci ${ }^{4}$ (D) \\ School of Medicine, University of Salerno Italy, 84084 Fisciano SA, Italy \\ 2 Unit of Dermosurgery Cutaneous Transplantations and Hard-to-HealWound, "Villa Fiorita" Private Hospital, 81031 \\ Aversa CE, Italy \\ 3 Institute for the Studies and Care of Diabetics, Abetaia, 81020 Casagiove CE, Italy \\ 4 Faculty of Medicine and Surgery, Vasile Goldis Western University of Arad, 310025 Arad, Romania \\ * Correspondence: alessandrocrisci@libero.it (A.C.);
}

Scopus Author ID 56125590000

Received: 8.08.2021; Revised: 20.09.2021; Accepted: 24.09.2021; Published: 16.10.2021

\begin{abstract}
We performed a study to evaluate the amount of Platelets and Leukocytes in a secondgeneration solid platelet concentrates in terms of Sensitivity, Specificity, Positive Predictive Value (VP+), Negative Predictive Value (VP-), False Negative Proportion, and False Positive Proportion of our Statistical Method. Blood was collected in anticoagulant-free PET tubes with silica for clot production and PRF membranes. The membranes and clots produced were examined. In a previous work, the authors, starting from the results obtained in Kitamura's work, wanted to elaborate a simpler and inexpensive system to calculate the exact amount of platelets and leukocytes contained in PRF, compared to the one present in whole blood, starting from a simple "haemochromocytometric examination". In this study, the authors have evaluated the Sensitivity, Specificity, Positive Predictive Value (VP+), Negative Predictive Value (VP-), False Negative Proportion, and False Positive Proportion of the "Statistical Method". Using the Statistical Method, we have that by reducing by $34.12 \%( \pm 28.2)$ the value of leukocytes obtained from the CBC examination. We obtained the value of leukocytes contained in the PRF membrane by t-PA digestion and by reducing by $15.12 \%( \pm 24.87)$ the value of platelets obtained by the same method, we obtained the value contained in solid platelet concentrates by t-PA digestion. The method thus proposed demonstrated a $\mathrm{Se}=0.75 ; \mathrm{Sp}=0.86$; $\mathrm{VP}+=0.75 ; \mathrm{VP}-=0.86$ for platelet counts, $\mathrm{Se}=0.47 ; \mathrm{Sp}=0.66 ; \mathrm{VP}+=0.80 ; \mathrm{VP}-=0.30$ for leukocyte counts. Conclusion: Our study sought to standardize the PRF preparation procedure by validating a statistical system to calculate the exact amount of platelets and leukocytes in second-generation solid platelet concentrates, making it easy to evaluate individual PRF arrays on time in the clinical setting. The Statistical method compared to the digestive method with t-PA for leukocyte and platelet counts demonstrated equal validity for platelets, but not so for leukocytes.
\end{abstract}

Keywords: blood derivatives; growth factors; leucocyte and platelets-rich fibrin; stem cells; statistical method.

(C) 2021 by the authors. This article is an open-access article distributed under the terms and conditions of the Creative Commons Attribution (CC BY) license (https://creativecommons.org/licenses/by/4.0/).

\section{Introduction}

Recently, "second-generation" Platelet-rich fibrin (PRF) was suggested as the novel bioregenerative therapeutic strategy to promote healing and soft tissue [1,2]. PRF or leukocyteand platelet-rich fibrin (L-PRF) is obtained from patients' blood and typically centrifuged at a relative spin force (RCF)-max/G-force of 800 for 12 minutes without any additives [3,4]. PRF 
not only functions as a three-dimensional fibrin scaffolding but also includes multiple autologous cells, such as platelets, macrophages, and neutrophils, and stem cells [5].

The ability of platelets to release substances from within a clot (PC-Platelet Concentrate) makes the latter a natural autologous source of growth factors and cytokines that can be used in the clinic to accelerate the physiological healing of a tissue injury $[1,2]$.

Because leukocytes, embedded neutrophils, and macrophages are in the top cell types present at injury sites, their function includes removing phagocytic fragments, bacteria, and necrotic tissue, thereby preventing infection. Neutrophils, along with platelets and, in association and their excreted growth promoters/cytokines, can facilitate tissue regeneration, generation of newly formed blood vessels (angiogenesis), with prevention of infection (bacteriostasis)[6].

Various types of second-generation concentrates have been produced by centrifuging blood drawn in different ways:

-L-PRF: $30 "$ of acceleration, 2' at $2700 \mathrm{rpm}, 4^{\prime}$ at $2400 \mathrm{rpm}, 3^{\prime}$ at $3000 \mathrm{rpm}$, and 36" of deceleration and stopping [1];

-Advanced-PRF: $1300 \mathrm{rpm}, 8$ minutes (189 g-forces) [7];

-Injectable-PRF: $700 \mathrm{rpm}$ (60 g-forces) per 3 minutes) [8];

- Horizontal-A-PRF: $1300 \mathrm{rpm}$ (189 g-forces) per 8 minutes [4];

Recently, the authors have studied an L-PRF, which appears to contain hematopoietic stem cells (HSCs) [5]. The presence of these HSC cells was detected mainly by immunohistochemical analysis to detect specific cellular markers CD34.

A recent study by Kitamura Y. et al. (2018) [9] shows a procedure for directly platelet count estimation in PRF. These authors utilized a professionally available method recombinant t-PA, Alteplase (GRTPA ${ }^{\circledR}$; Mitsubishi Tanabe Pharma Corp., Osaka, Japan) via a digestion method. Using this fairly elaborate procedure, they demonstrated that t-PA is sufficiently potent to succeed in counting dispersed platelets aggregated in platelet-enriched insoluble fibrin matrices (Table 1).

Table 1. Comparison of Method for Count with t-PA digestion and CBC for WBC and PLT [9-10, 16].

\begin{tabular}{|c|c|c|c|c|c|c|c|}
\hline \multicolumn{4}{|c|}{ WBC conta $\times 10^{\wedge 5} / \mathrm{CGF}$} & \multicolumn{4}{|c|}{ PLT conta $\times 10^{\wedge} 7 / \mathrm{CGF}$} \\
\hline $\begin{array}{c}\text { t-PA- } \\
\text { digerito }\end{array}$ & $\begin{array}{c}\text { W.B. } \\
\text { ematico }\end{array}$ & $\begin{array}{l}\text { W.B- } \\
\text { t-PA }\end{array}$ & $\begin{array}{c}\text { W.B. } \\
- \\
34,12 \pm 28.2 \%\end{array}$ & $\begin{array}{c}\text { t-PA- } \\
\text { digerito }\end{array}$ & $\begin{array}{c}\text { W.B. } \\
\text { ematico }\end{array}$ & $\begin{array}{c}\text { W.B-t- } \\
\text { PA }\end{array}$ & $\begin{array}{c}\text { W.B. } \\
- \\
15,12 \pm 24.87 \%\end{array}$ \\
\hline 136.50 & 355.60 & 61.61 & 234.27 & 108.60 & 157.48 & 31.04 & 133.67 \\
\hline 242.60 & 331.80 & 26.88 & 218.59 & 107.10 & 89.27 & -19.97 & 75.77 \\
\hline 314.00 & 400.00 & 21.50 & 263.52 & 91.10 & 100.80 & 9.62 & 85.56 \\
\hline 310.00 & 376.00 & 17.55 & 247.71 & 90.20 & 116.80 & 22.77 & 99.14 \\
\hline 312.00 & 583.20 & 46.50 & 384.21 & 132.80 & 261.63 & 49.24 & 222.07 \\
\hline 184.00 & 287.00 & 35.89 & 189.08 & 81.70 & 152.60 & 46.46 & 129.53 \\
\hline 112.20 & 249.80 & 55.08 & 164.57 & 126.20 & 141.75 & 10.97 & 120.32 \\
\hline 431.80 & 342.00 & -26.26 & 225.31 & 217.80 & 190.76 & -14.17 & 161.92 \\
\hline 451.50 & 343.20 & -31.56 & 226.10 & 251.10 & 197.34 & -27.24 & 167.50 \\
\hline 205.00 & 378.00 & 45.77 & 249.03 & 76.30 & 96.60 & 21.01 & 81.99 \\
\hline 401.30 & 337.50 & -18.90 & 222.35 & 217.10 & 171.00 & -26.96 & 145.14 \\
\hline 112.20 & 350.00 & 67.94 & 230.58 & 126.20 & 155.00 & 18.58 & 131.56 \\
\hline 136.50 & 426.30 & 67.98 & 280.85 & 108.60 & 132.50 & 18.04 & 112.47 \\
\hline 242.60 & 380.00 & 36.16 & 250.34 & 107.10 & 117.80 & 9.08 & 99.99 \\
\hline 401.30 & 667.30 & 39.86 & 439.62 & 217.10 & 228.40 & 4.95 & 193.87 \\
\hline 431.80 & 616.00 & 29.90 & 405.82 & 217.80 & 245.60 & 11.32 & 208.47 \\
\hline 451.50 & 592.80 & 23.84 & 390.54 & 251.10 & 248.50 & -1.05 & 210.93 \\
\hline 312.00 & 563.20 & 44.60 & 371.04 & 132.80 & 126.70 & -4.81 & 107.54 \\
\hline 314.00 & 410.80 & 23.56 & 270.64 & 91.10 & 104.60 & 12.91 & 88.78 \\
\hline 184.00 & 420.80 & 56.27 & 277.22 & 81.70 & 100.70 & 18.87 & 85.47 \\
\hline 126.50 & 385.40 & 67.18 & 253.90 & 34.10 & 108.20 & 68.48 & 91.84 \\
\hline
\end{tabular}




\begin{tabular}{|c|c|c|c|c|c|c|c|c|}
\hline \multicolumn{4}{|c|}{ WBC conta $\times 10^{\wedge} 5 / \mathrm{CGF}$} & & \multicolumn{4}{|c|}{ PLT conta $\times 10^{\wedge} 7 / \mathrm{CGF}$} \\
\hline $\begin{array}{c}\text { t-PA- } \\
\text { digerito }\end{array}$ & $\begin{array}{c}\text { W.B. } \\
\text { ematico }\end{array}$ & $\begin{array}{l}\text { W.B- } \\
\text { t-PA }\end{array}$ & $\begin{array}{c}\text { W.B. } \\
- \\
34,12 \pm 28.2 \% \\
\end{array}$ & & $\begin{array}{c}\text { t-PA- } \\
\text { digerito }\end{array}$ & $\begin{array}{c}\text { W.B. } \\
\text { ematico }\end{array}$ & $\begin{array}{l}\text { W.B-t- } \\
\text { PA }\end{array}$ & $\begin{array}{c}\text { W.B. } \\
- \\
15,12 \pm 24.87 \% \\
\end{array}$ \\
\hline 205.00 & 469.20 & 56.31 & 309.11 & & 76.30 & 136.60 & 44.14 & 115.95 \\
\hline 290.40 & 462.00 & 37.14 & 304.37 & & 80.40 & 144.90 & 44.51 & 122.99 \\
\hline 274.29 & 422.95 & 34.12 & 278.64 & Mean & 131.49 & 153.28 & 15.12 & 130.11 \\
\hline 115.31 & 110.75 & 28.20 & 72.96 & $\pm \mathrm{D} . \mathrm{S}$ & 63.45 & 52.26 & 24.87 & 44.36 \\
\hline
\end{tabular}

Recently, AA. (2019) have elaborated, in collaboration with Kawase's Japanese group, a statistically valid mathematical method to predict the presence of cells in PRF starting from the performance of a blood thrombocytometric examination (Table 1) the validation of a simple and costless method to quantify the exact amount of platelets \& leukocytes contained in platelet concentrates [10].

\section{Material and Methods}

Starting from the study by Kitamura Y. et al.(2018)[9], the AAs aimed to: evaluate Se (Sensitivity), Sp (Specificity), VP+ (Positive Predictive Value), VP- (Negative Predictive Value), False-Negative Proportion, and False-Positive Proportion of the two methods (digestion with t-PA and Statistic) compared (Table 2).

Table 2. Applied tests on statistical evaluation of cell count.

Proportion False Negatives = It is the fraction of test negatives

Proportion False Positives $=$ It is the fraction of test positives

Sensitivity $($ If $)=$ Indicates the odds of obtaining a true positive result

Specificity $(\mathrm{Sp})=$ Indicates the probability of obtaining a false-positive result

Positive Predictive Value $(V P+)=$ Indicates the odds that a test + is actually positive (True Positive)

Negative Predictive Value $(V P-)=$ Indicates the probability that a test - is actually negative (True Negative)

Blood was collected in tubes without anticoagulant or PET gel separator with silica for clot production and PRF membranes (Vacuaptaca clot activator tubes cod.30023).

When PRF preparation protocols are described in the literature, very often there are no indications of important parameters (e.g., if anticoagulant is used and its volume, volume of blood collected and final PDP reached, working temperature, the time elapsed between collection/processing/analysis of samples), which leads to questionable and not comparable results.

In our protocol, blood was rapidly collected with a $19 \mathrm{G}$ needle in $9 \mathrm{cc}$ vacutainer tubes using a vacuum system (average collection rate of 22", less than 25 " per tube) and immediately (within 1 minute) centrifuged according to the above description at a temperature above $21^{\circ} \mathrm{C}$.

Adopted processing temperature range $21.3 / 22.5^{\circ} \mathrm{C}$.

Cold processing $\left(<20^{\circ} \mathrm{C}\right)$ of clots and membranes leads to impoverishment of the biological and physical characteristics of the product, and the ideal minimum temperature for storage for some hours is $+4^{\circ} \mathrm{C}$ (no more than 2 hours).

In addition, this was not designed as a clinical study, and the control group is represented by the results obtained with the count with t-PA digestion.

A blood sample was also collected to execute a blood enumeration utilizing K3E 5.4 mg tubes with EDTA (VacuMed). Leukocyte and platelet concentrations of L-PRF were analyzed with a CBC in standard bays and performed with a Cell Dyn $3500 \mathrm{R}$ cell counter (Abbott Laboratories; Abbott Park, IL, USA).

AA., in a recent study [10], elaborating the data obtained by Kawase's study group [9] obtained that, by reducing by $15.12 \%$ the importance of the platelet counting from the 
Hemochrome and by $34.12 \%$ the importance of the leukocyte count, they derived the value obtained by the digestion method with t-PA [9] with a much simpler system. Therefore, they experimented with an easy and economical system to indirectly calculate the number of platelets and leukocytes contained in self-compressed platelet concentrates (not liquid). The study has shown that it is possible to estimate the number of cells in solid biomaterials, a complex cellular system for the presence of platelets, leukocytes, stem cells, etc., using a clinical method applicable in a rapid (max 15 minutes) and safe way (Statistical Method). As confirmed in the current research study, the authors wanted to review the results by evaluating the parameters of Sensitivity, Specificity, Positive Predictive Value, Negative Predictive Value of the Statistical Method, comparing them with the same parameters obtained with the Method of Digestion with t-PA.

\subsection{Statistical measurements and analysis.}

Data elements were investigated using version 6.0 of the Santon-Glantz 2007 Statistical Package for Biomedical Disciplines. Sensitivity and Specificity for platelet and leukocyte counts were calculated on the data obtained with t-PA by the Japanese Group (Aureo test) and on the data obtained with the "Statistical Method" by AA (WB-15.12\% for platelets and WB$34.12 \%$ for $\mathrm{WBC}$ ), considering as cut-off the average value of count with t-PA digestion (131.49 vs. 274.29). In addition, Positive and Negative Predictive Values (VP+, VP-) and the Proportion of False Negatives and Positives were calculated on the same results (Table 2).

The validity and accuracy of the measurements were assessed by the coefficient of variation, considering the values valid if it is $<2 \%$ over at least three measurements.

The zero hypothesis $\left(\mathrm{H}_{\varnothing}\right)$ formulated is that there is no statistically significant difference between values measured by "t-PA method" and values measured by the "blood count method" (W.B.) \pm X\%, for both PLT and WBC.

$$
\text { Formally } \mathrm{H}_{\varnothing}: \mathrm{t}-\mathrm{PA}=\mathrm{W} . \mathrm{B} .(\mathrm{PLT})(\mathrm{WBC}) \pm \mathrm{X} \% \text {; }
$$

\section{Results and Discussion}

The membrane and clot production procedure was well tolerated in all examined subjects.

No significant differences were detected in the baseline hematology comparison of the examined subjects, which had a mean $\mathrm{RBC}$ concentration of $5.6 \times 10^{6} / \mathrm{mL}( \pm 1.1$ I.C.95 $\%)(\mathrm{p}=0.34)\left(\right.$ range: $\left.4-6 \times 10^{6} / \mathrm{mL}\right)$, of WBC of $5.1 \times 103 / \mathrm{mL}( \pm 0.37$ I.C.95\% $)(\mathrm{p}=0.24)($ range:4.3$10 \times 103 / \mathrm{mL})$ and a Mean Platelet Count of $176.8 \times 10^{3} / \mathrm{mL}( \pm 15.3$ I.C.95\% $)(\mathrm{p}=0.15)$ (range: $150-350 \times 10^{3} / \mathrm{mL}$ ).

The values were compared with the Statistical Indirect Count Method.

In a suggestive phase of the experimentation, we compared the platelet and leukocyte count method with the t-PA digestion proposed by Kawase [9] with the Statistical method proposed in 2019 [10], whose values are shown in Table 1. In this study, we were able to show that, by reducing by $15.12 \%( \pm 24.8)$ the amount of the platelet counting to the amount of the $\mathrm{CBC}$, and by $34.12 \%( \pm 28.2)$ the amount of the leukocyte counting to the amount of the CBC, we will obtain the value obtained with the method of digestion with t-PA.

We constructed the values obtained with the two methods, then the ROC (Relative Operating Characteristic) curve for PTL and WBC for values obtained with t-PA dig and with W.B.-15.12\% for PLT and $-34.12 \%$ for WBC. 
The cut-off considered is represented by the average t-PA for PLT (131.49) and WBC (274.29). (Figure 1 A-B).
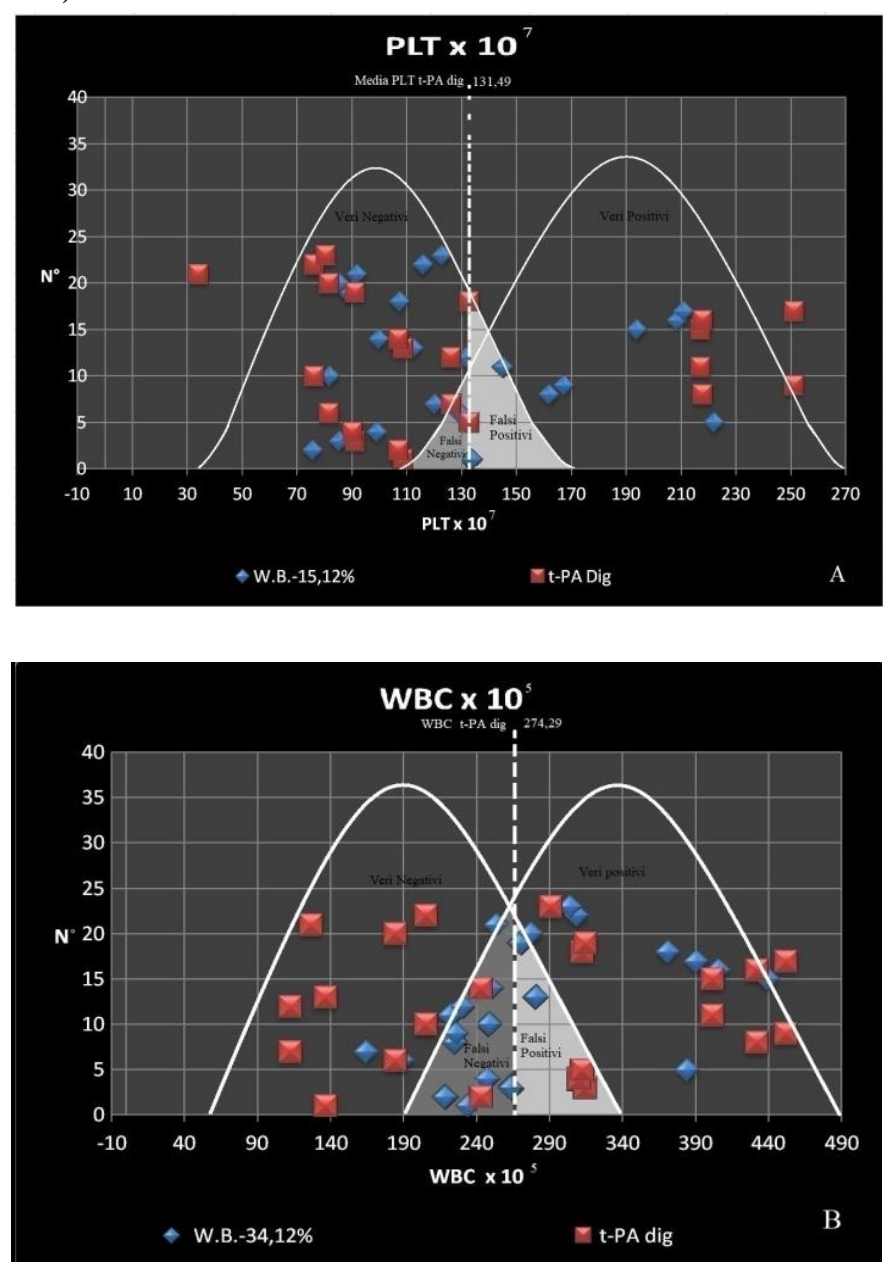

Figure 1. ROC curve for (A) PTL and (B) WBC for values obtained with t-PA dig and with W.B.-15.12\% for PLT and $-34.12 \%$ for WBC. The cut-off is represented by the mean t-PA for PLT (131.49) and for WBC

(274.29).

On these values, we calculated, in the simplest hypothesis in which it is assumed that the "gold test" provides results perfectly corresponding to the truth, the Sensitivity (Se), the Specificity (Sp), the Positive Predictive Value $(V P+)$, the Negative Predictive Value $(V P-)$, the Proportion of False Negatives and the Proportion of False Positives (Tables 2, 3 and 4). We have therefore assumed as a "gold test" the one resulting from the t-PA dig count.

Table 3. Contingency table for PLT. The "Gold Standard" was referred to as the t-PA dig test.

PLT Aureus Test (t-PA dig)

\begin{tabular}{|c|c|c|c|c|c|c|}
\hline & M. & N.M. & Tot. & Off Scale & & \\
\hline Pos & 4 & 2 & 6 & 0 & $S e=0.80$ & $V P+=0.66$ \\
\hline $\mathrm{Neg}$ & 1 & 13 & 14 & 0 & $S p=0.86$ & $V P-=0.92$ \\
\hline Tot & 5 & 15 & 23 & 3 & Prop.False Neg $=0.2$ & Prop.False Pos $=0.13$ \\
\hline \multicolumn{7}{|c|}{ Test PLT (WB-15.12\%) } \\
\hline & M. & N.M. & Tot. & Off Scale & & \\
\hline Pos & 6 & 2 & 8 & 0 & $S e=0.75$ & $V P+=0.75$ \\
\hline $\mathrm{Neg}$ & 2 & 13 & 15 & 0 & $S p=0.86$ & $V P-=0.86$ \\
\hline Tot & 8 & 15 & 23 & 0 & Prop.False $\mathrm{Neg}=0.25$ & Prop.False Pos $=0.13$ \\
\hline
\end{tabular}


Table 4. WBC contingency table. The "Gold Standard" was referred to as be the t-PA dig test.

Golden test WBC (t-PA dig).

\begin{tabular}{|c|c|c|c|c|c|c|}
\hline & M. & N.M. & Tot. & Off Scale & & \\
\hline Pos & 8 & 3 & 11 & 0 & $S e=0.80$ & $V P+=0.75$ \\
\hline $\mathrm{Neg}$ & 2 & 9 & 11 & 0 & $S p=0.75$ & $V P-=0.81$ \\
\hline Tot & 10 & 12 & 23 & 1 & Prop.False $\mathrm{Neg}=0.2$ & Prop.False Pos $=0.25$ \\
\hline \multicolumn{7}{|c|}{ Test WBC (WB-34.12\%) } \\
\hline & M. & N.M. & Tot. & Off Scale & & \\
\hline Pos & 8 & 2 & 10 & 0 & $S e=0.47$ & $V P+=0.80$ \\
\hline Neg & 9 & 4 & 13 & 0 & $S p=0.66$ & $V P-=0.30$ \\
\hline Tot & 17 & 6 & 23 & 0 & Prop.False Neg $=0.53$ & Prop.False Pos $=0.33$ \\
\hline
\end{tabular}

The results obtained showed for PLT a Sensitivity of 0.75 (Gold Standard=0.80), Specificity of 0.86 (Gold Standard=0.86), for WBC a Sensitivity of 0.47 (Gold Standard=0.80), Specificity of 0.66 (Gold Standard=0.75).

Also a $V P+$ of 0.75 (Gold Standard=0.66), $V P$ - of 0.86 (Gold Standard=0.92) for PLT. A $V P+$ of 0.80 (Gold Standard=0.75), VP- of 0.30 (Gold Standard=0.81) for WBC. The Proportion of False Negatives is 0.25 for PLT (Gold Standard=0.2) and 0.53 for WBC (Gold Standard=0.2). The Proportion of False Positives is 0.13 for PLT (Gold Standard=0.13) and 0.33 for WBC (Gold Standard=0.25).

The PRF, the group, adapts to the needs of a diversity of surgeries procedures. Like clots and membranes, PRF has a shape and volume that is simple to associate with most other surgical procedures, including the following filling and interposition of healing biomaterials or as protective membranes for injury treatment. Finally, it is easy to prepare in medium quantities and inexpensive, making it suitable for everyday clinical practice. It was used to benefit humans by AA., particularly in treating diabetic skin ulcers, including chronic osteomyelitis [11-13]. The study performed by McLellan et al.[14] demonstrated that equine PRF, similar to human PRF, is an instantaneous and sustained resource of tissue growth factors. Our trial sought to confirm the validation of the "Statistical Method" by standardizing the procedure for the preparation of L-PRF, which, while remaining an easy technique to perform at low cost and requiring no specialized equipment, has some consolidation in the generation of an L-PRF membrane.

L-PRF and its derivatives represent tremendous critical progress in the evolving platelet concentrates. It is essentially a fibrin matrix membrane containing platelets and leukocytes entrapped in combination with stem cells. These rigid films exhibit exceptional handling properties and can be sutured securely into an anatomically desired position In open surgery procedures.

Although, the physical and biological characteristics are virtually unknown and have yet to be fully investigated.

Based on these results, it is evident that L-PRF is a revolutionary new bio-material with unique features: -foreseeable preparation from self blood; -simple protocol; -defined architecture; -impressive mechanical properties; -and plenty of growth promoter factors derived from activated platelets.

Our tests performed on equine blood are undoubtedly capable of improving the comprehension of mechanisms of healing and advancing the field of personalized medicine.

Their influence should be studied with particular attention concerning leukocyte count and concentration, as their presence or absence could explain the conflicting results noted in multiple trials [15-22]. 
The results obtained in this study on the sustainability of the "Statistical" counting of platelets and leukocytes showed for PLT a Sensitivity of 0.75 (Gold Standard=0.80), Specificity of 0.86 (Gold Standard=0.86), for WBC a Sensitivity of 0.47 (Gold Standard=0.80), Specificity of 0.66 (Gold Standard=0.75).

\section{Conclusions}

Concluding this research, we can state that the preparation of PDPs is extremely technically challenging due to the delicate nature of platelets that are frequently initiated during the procedure. Consequently, special considerations are needed to maximize the therapeutic benefits of these blood products. Today, their procurement is supported by the poorly controlled bulk release. As a result, prolonged treatments require multiple treatments. This results in large fluctuations in Platelet, Leukocyte, and Stem Cell concentrations, compromising clinical predictability. Hence the need to experiment with simple methods for counting, even indirectly, contained cells, as biomaterials can act as controlled-release devices, allowing prolonged or even on-demand administration of these cocktails with the addition of growth factors.

This experimentation indicates it is useful to use the WB value of PLT- $15.12 \%$ to obtain the values of count for t-PA with sufficient statistical accuracy with a Sensitivity of 0.75 vs. 0.80 and a Specificity of 0.86 vs. 0.80 , therefore very similar to the "Gold Test". Using instead, the value of WB of WBC- $34.12 \%$ has a Sensitivity and Specificity much lower than the "Gold Standard" (Sensitivity of 0.47 vs. 0.80, Specificity of 0.66 vs 0.75 ).

\section{Funding}

This research received no external funding.

\section{Acknowledgments}

I want to thank the operating room nurses and the owner of the "Casa di Cura" Villa Fiorita in Aversa (Italy) for their collaboration.

\section{Conflicts of Interest}

The authors declare no conflict of interest.

\section{References}

1. Crisci, A.; Benincasa, G.; Crisci, M.; Crisci, F. Leukocyte Platelet-Rich Fibrin (L-PRF), a new biomembrane useful in tissue repair: basic science and literature review. Biointerface Res Appl Chem 2018, 8, 3635-43.

2. Pretorius, E. Ultrastructural comparison of the morphology of three different platelet and fibrin fiber preparations, The Anatomical Record 2007, 290, 188-198.

3. Hasan, F.K. Characterization of leukocyte-platelet rich Fibrin, a novel biomaterial, 2015, http://scholarscompass.vcu.edu/etd.

4. Miron, R.J.; Chai, J.; Zheng, S.; Feng, M.; Sculean, A.; Zhang, Y. A Novel Method for Evaluating and Quantifying Cell Types in Platelet Rich Fibrin and an Introduction to Horizontal Centrifugation, J Biomed Mater Res 2019, 107, 2257-2271, https://dx.doi.org/10.1002/jbm.a.36734.

5. Crisci, A.; Barillaro, M.C.; Lepore, G.; Cardillo, F. L-PRF Membrane (Fibrin Rich in Platelets and Leukocytes) and Its Derivatives (A-PRF, i-PRF) are Helpful as a Basis of Stem Cells in Regenerative Injury Treatment: Trial Work on the Horse. International Blood Research \& Reviews 2020, 10, 1-14, https://doi.org/10.9734/ibrr/2019/v10i230117. 
6. $\quad$ Feng, M.; Wang, Y.; Zhang, P.; Zhao, Q.; Yu, S.; Shen, K.; Miron, R.J.; Zhang, Y. Antibacterial effects of platelet-rich fibrin produced by horizontal centrifugation. Int $J$ Oral Sci 2020, 12, 32, https://doi.org/10.1038/s41368-020-00099-w.

7. Ghanaati, S.; Booms, P.; Orlowska, A. et al. Advanced Platelet-Rich Fibrin: a new concept for cell- based tissue engineering by means of inflammatory cells. J Oral Implantol 2014, 40, 679-89.

8. Schär, M.O.; Diaz-Romero, J.; Kohl, S.; Zumstein, M.A.; Nesic, D. Platelet-rich Concentrates Differentially Release Growth Factors and Induce Cell Migration In Vitro. Clin. Orthop. Relat. Res. 2015, 473, 1635-43, http://dx.doi.org/10.1007/s11999-015-4192-2.

9. Kitamura, Y.; Watanabe, T.; Nakamura, M.; Isobe, K.; Kawabata, H.; Uematsu. K.; Okuda, K.; Nakata, K.; Tanaka, T.; Kawase, T. Platelet Counts in Insoluble Platelet-Rich Fibrin Clots: A Direct Method for Accurate Determination, Front Bioeng Biotechnol 2018, 6, 4, http://dx.doi.org/10.3389/fbioe.2018.00004.

10. Crisci, A.; Kawase, T.; D'Adamo, R.; Crisci, M. Experimental research on a technique for quantification of platelets and leukocytes in second-generation platelet concentrates, International Journal of Current Medical and Pharmaceutical Research 2019, 05, 4792-99, http://dx.doi.org/10.24327/23956429.ijcmpr201912804.

11. Crisci, A.; Marotta, G.; Licito, A.; Serra, E.; Benincasa, G.; Crisci, M. Use of leukocyte platelet (L-PRF) rich fibrin in diabetic foot ulcer with osteomyelitis (three clinical cases report), Diseases 2018, 6, 30, http://dx.doi.org/10.3390/diseases6020030.

12. Crisci, A.; D'Adamo, R.; Crisci, M. The Second-Generation Platelet Concentrates in The Treatment of Chronic Ostemyelitis: One Modern Regenerative Surgery. International Journal of Research GRANTHAALAYAH, 2020, 8, 112-122, https://doi.org/10.29121/granthaalayah.v8.i10.2020.1842.

13. Giuliano, G.; Crisci, M.; D’Adamo, R.; Crisci, A. I concentrati piastrinici di seconda generazione nella terapia dell'osteomielite cronica: una chirurgia rigenerativa moderna, Giornale Italiano di Ortopedia e Traumatologia 2020, 46, 1-12, https://doi.org/10.32050/0390-0134-271.

14. McLellan, J.; Plevin, S.; Temporal release of growth factors from platelet-rich fibrin (PRF) and platelet-rich Plasma (PRP) in the horse: a comparative in vitro analysis. Intern J Appl Res Vet Med 2014, 1, 48-57.

15. Sato, A.; Kawabata, H.; Aizawa, H.; Tsujino, T.; Isobe, K.; Watanabe, T.; Kitamura, Y.; Miron, J.R.; Kawase, T. Distribution and quantification of activated platelets in platelet-rich fibrin matrices, Platelets 2020, https://doi.org/10.1080/09537104.2020.1856359.

16. Crisci, A.; Kawase. T.; D’Adamo, R.; Crisci, M. Quantificazione delle piastrine e dei leucociti nei concentrati piastrinici solidi del sangue per uso chirurgico: studio di una tecnica sperimentale. Chirurgia 2021, 34, https://doi.org/10.23736/S0394-9508.20.05129-3.

17. Crisci, A. New Platelet Concentrates Useful in Tissue Repair. Platelet-rich Fibrin with Leukocytes $(L-P R F)$, Advanced Platelet-Rich Fibrin (A-PRF) and Injectable Plateletrich Fibrin ( $i$-PRF), BP International Edition, United Kingdom, 2021, https://doi.org/10.9734/bpi/mono/978-93-91473-15-0.

18. Crisci, A.; Kawase, T.; D’Adamo, R.; Crisci, M. Utilità dei derivati del sangue di seconda generazione nella terapia rigenerativa delle ferite complesse delle estremità. Med Chir Caviglia Piede 2020, 44, 3-13, https://doi.org/10.23736/S2284-2993.20.01811-7.

19. Crisci, A.; Conte A. The membranes of $L$-PRF (fibrin rich in platelets and leukocytes) and its derivatives (A-PRF, $i$-PRF): Use as a source of stem cells in regenerative wound therapy in horses, British Journal of Medical \& Health Sciences (BJMHS) 2020, 2, 329-336.

20. Crisci, A.; Minniti, C.A.; Conte, A.; Crisci, M.; Cardillo F. Second Generation Platelet Concentrates - LPRF (Fibrin Rich in Platelets and Leukocytes) and Its Derivatives (A-PRF, i-PRF)-: Morphological Characteristics to be Used in Modern Regenerative Surgery. Experimental Research. J Clin Haematol 2020, 1, 90-102, https://doi.org/10.33696/haematology.1.014.

21. Crisci, A.; D'Adamo, R.; Crisci, M. Clinical Trial on Solid Second-Generation Platelet-Concentrates in the Management of the Chronic Osteomyelitis: Advanced Bioregenerative Surgeries, The Intern. Journal of Lower Extremity Wounds 2021, 1-11, https://doi.org/10.1177/15347346211003732.

22. Crisci, A. La rigenerazione dei tessuti con Fibrina Ricca di Piastrine: i Nuovi concentrati piastrinici, Aracne Editrice, Roma 2021. 